\title{
Progress in Vaccination of Prophylactic Human Papillomavirus Vaccine
}

\author{
Xu Zhou ${ }^{1}$, Lihua Sun ${ }^{2}$, Xiaoxiao Yao ${ }^{2}$, Guangquan $L^{2}{ }^{2}$, Yicun Wang ${ }^{2}$ and Yang Lin ${ }^{1 *}$ \\ ${ }^{1}$ Department of Gynecology and Obstetrics, The Second Hospital of Jilin University, Changchun, China, ${ }^{2}$ Jilin Provincial Key \\ Laboratory on Molecular and Chemical Genetic, The Second Hospital of Jilin University, Changchun, China
}

The human papillomavirus (HPV) vaccine plays an important role in preventing a series of diseases caused by HPV. Recent studies have shown that as a primary prevention measure, it can considerably prevent HPV infection and HPV-associated cervical cancer. However, studies on the safety, efficacy, and coverage of the HPV vaccine remain insufficient, especially in developing countries. Therefore, in this review, we outlined the recent studies of the HPV vaccine in terms of immunogenicity, safety, efficacy, latest vaccination concepts, and strategies. This review may provide a theoretical basis for use of the HPV vaccine.

Keywords: HPV, vaccine, effectiveness, safety, implementation

\section{OPEN ACCESS}

Edited by:

Suzanne Garland,

Royal Women's Hospital, Australia

Reviewed by:

Rupsa Basu,

TechnoVax Inc., United States

David Pejoski,

Université de Genève, Switzerland

*Correspondence:

Yang Lin

linyang1155@163.com

Specialty section:

This article was submitted to Vaccines and Molecular Therapeutics, a section of the journal

Frontiers in Immunology

Received: 25 December 2019

Accepted: 03 June 2020

Published: 10 July 2020

Citation:

Zhou X, Sun L, Yao X, Li G, Wang Y and Lin $Y(2020)$ Progress in Vaccination of Prophylactic Human

Papillomavirus Vaccine.

Front. Immunol. 11:1434

doi: 10.3389/fimmu.2020.01434

\section{INTRODUCTION}

One of the malignant tumors with the highest prevalence among women includes cervical cancer. According to the World Health Organization (WHO), there are 570,000 cases and 313,000 deaths added yearly worldwide (1). In the 1880s, German scientist Zur et al. found that correlation between human papillomavirus (HPV) infection and cervical cancer (2). Prophylactic HPV vaccine plays an important role in reducing HPV infection rate, which is a revolutionary step in preventing HPV-related diseases, particularly cervical cancer.

Since 2006, the prophylactic HPV vaccine has been licensed in over 100 countries (3). It is currently available in three types (Table 1): Cervarix (GlaxoSmithKline Biologicals, Belgium), Gardasil (Merck \& Co., USA) and Gardasil9 (Merck \& Co., USA) (4). Cervarix is a 2-valent HPV (2vHPV) vaccine has two virus-like particles (VLPs) including HPV 16 and 18 VLPs, which causes $70 \%$ of cervical malignancies (5). Gardasil is a 4-valent HPV (4vHPV) vaccine containing HPV 16 and 18 VLPs, and type 6 and 11 VLPs, which is associated with $90 \%$ of genital wart infections (6). Gardasil 9 is a 9-valent HPV (9vHPV) vaccine for HPV 6/11/16/18/31/33/45/52/58. In 2007, 4vHPV, and 2vHPV were licensed (Figure 1). In 2014, 9vHPV was licensed (7). Currently, vaccination can reduce the incidence of female and male reproductive tract diseases has been determined, including anal and oral HPV infection and cervical, vaginal, vulvar, penile, and anal intraepithelial neoplasia (4). Since the license was granted, HPV infection and incidence rates have dropped considerably worldwide. The safety, efficacy, and duration of prophylactic HPV vaccine have been confirmed by the WHO, which makes vaccination possible to control the occurrence of HPV-associated cervical cancer in humans. This paper reviews the latest progress of three kinds of HPV vaccines and the prospective vaccines. It will provide a theoretical basis for further application of the HPV vaccine.

HPV is an epitheliophilic virus belonging to the papillomavirus family and has a variety of hosts of animals and humans (8). On the basis of the ability of HPV to cause lesions, it is divided 
TABLE 1 | Characteristics of HPV vaccines.

\begin{tabular}{|c|c|c|c|}
\hline Name & Cervarix (2vHPV vaccine) & Gardasil (4vHPV vaccine) & Gardasil 9 (9vHPV vaccine) \\
\hline Antigen & L1 VLP of HPV 16 and 18 & L1 VLP of HPV $6,11,16$, and 18 & $\begin{array}{l}\text { L1 VLP of HPV } 6,11,16,18,31,33,45 \\
52 \text {, and } 58\end{array}$ \\
\hline Dose of L1 VLP types & 20 and $20 \mu \mathrm{g}$ & $20,40,40$, and $20 \mu \mathrm{g}$ & $30,40,60,40,20,20,20,20$, and $20 \mu \mathrm{g}$ \\
\hline Adjuvant & $\begin{array}{l}500 \mu \mathrm{g} \text { alluminium hydroxide, } 50 \mu \mathrm{g} \\
\text { 3-O-deacylated-4'-monophosphoryl } \\
\text { lipid A }\end{array}$ & $\begin{array}{l}225 \mu \mathrm{g} \text { alluminium hydroxyphosphate } \\
\text { sulfate }\end{array}$ & $\begin{array}{l}500 \mu \mathrm{g} \text { alluminium hydroxyphosphate } \\
\text { sulfate }\end{array}$ \\
\hline System & $\begin{array}{l}\text { BEVS, baculovirus expression vector } \\
\text { system }\end{array}$ & Yeast & Yeast \\
\hline Producer cells & $\begin{array}{l}\text { Trichoplusia ni }(\mathrm{Hi} \mathrm{5)} \text { insect cell line } \\
\text { infected with L1 recombinant } \\
\text { baculovirus }\end{array}$ & $\begin{array}{l}\text { Saccharomyces cerevisiae (baker's yeast) } \\
\text { expressing L1 }\end{array}$ & $\begin{array}{l}\text { Saccharomyces cerevisiae (baker's yeast) } \\
\text { expressing L1 }\end{array}$ \\
\hline Sodium chloride, mg & 4.4 & 9.56 & 9.56 \\
\hline L-Histidine, mg & & 0.78 & 0.78 \\
\hline Polysorbate $80, \mu \mathrm{g}$ & & 50 & 50 \\
\hline Sodium borate, $\mu \mathrm{g}$ & & 35 & 35 \\
\hline $\begin{array}{l}\text { Sodium dihydrogen phosphate } \\
\text { dihydrate, mg }\end{array}$ & 0.624 & & \\
\hline Party & GlaxoSmithKline & Merck & Merck \\
\hline Approval year & 2007: licensure by Australia and EU & 2006: licensure by FDA & 2014: licensure by FDA \\
\hline Indications & $\begin{array}{l}\text { Females: Cervical precancer and } \\
\text { cancer } \\
\text { Males: Not approved for use in males }\end{array}$ & $\begin{array}{l}\text { Females: Cervical precancer and cancer; } \\
\text { Genital warts } \\
\text { Males: Anal precancer and cancer; } \\
\text { Genital warts }\end{array}$ & $\begin{array}{l}\text { Females: Cervical precancer and cancer; } \\
\text { Genital warts } \\
\text { Males: Anal precancer and cancer; } \\
\text { Genital warts }\end{array}$ \\
\hline Vaccination schedule & 0,1 , and 6 months & 0,2 , and 6 months & 0,2 , and 6 months \\
\hline
\end{tabular}

HPV, human papillomavirus; VLP, virus-like particle.2vHPV, 2-valent human papillomavirus; 4vHPV, 4-valent human papillomavirus; 9vHPV, 9-valent human papillomavirus.

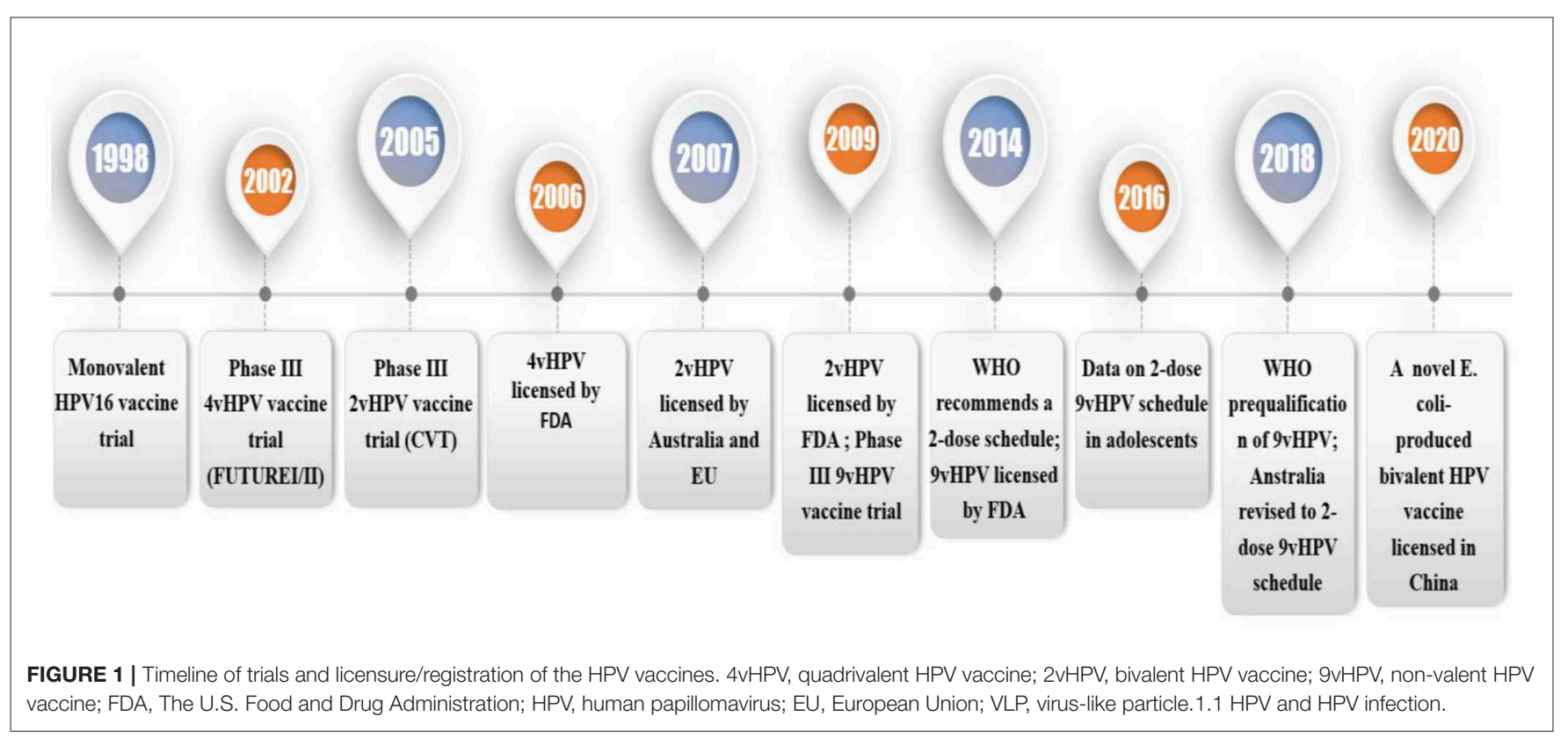

into two types: high-risk and low-risk types, among which the high-risk type can cause epithelial precancerous lesions and cancer (9). HPV16, 18, 31, 33, 35, 39, 45, 51, 52, 56, 58, 59, and 68 constitute 13 types of high-risk HPV. HPV 16 and HPV 18 have the highest carcinogenicity (5).
HPV may cause cervical, vulvar, vaginal, penile, anal, rectal, and oropharyngeal cancers, which account for more than $80 \%$ of HPV-related cancers in the cervix (10). The highest rate of genital HPV infection occurs in women younger than 30 who are sexually active. Eighty to ninety percent of women have at 


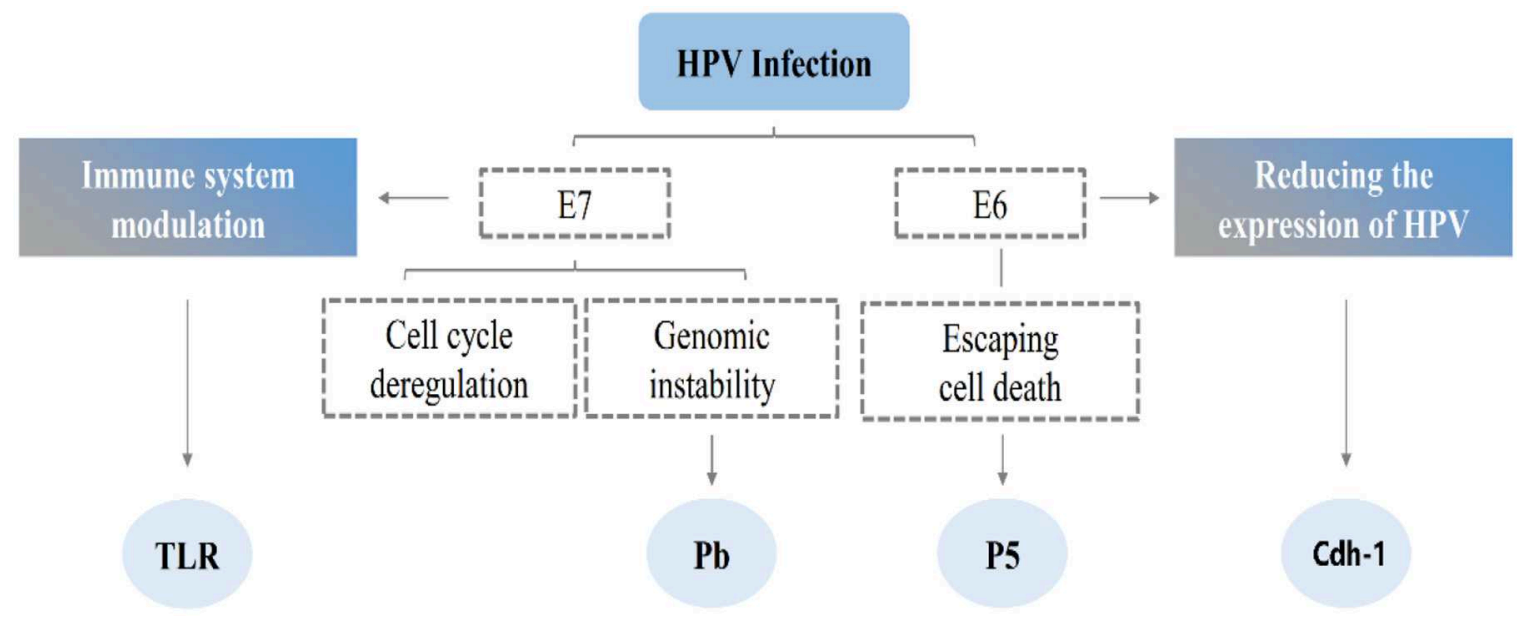

FIGURE 2 | The possible mechanism of HPV infection. Simultaneous molecular targets of the high-risk E6 and E7 oncoprotein. E6 and E7 are known to interact with a diverse range of molecules that are involved in several cellular pathways namely in immune system modulation, invasion, cell cycle deregulation. E6 and E7 share common targets that participate in important processes of cell invasion and inflammation, this cooperation may result in a synergistic mechanism, thereby promoting malignant cell transformation. HPV, human papillomavirus; INF, interferon; TLRs, toll-like receptors; Cdh-1, E-cadherin gene.

least one HPV infection after sex, although the vast majority are asymptomatic and transient infections that can be cleared within 6-18 months. According to statistics, the HPV infection rate is $30 \%$ in the first year after the first instance of sexual behavior and reaches $50 \% 3$ years later (11).

The HPV infection is limited to basal epithelial cells, which are infected by micro-scratches on the epithelial surface, which enter the basal cells of the cells with wound healing (12). Thereafter, the DNA of the virus is released from the capsid and enters the nucleus in a free form. In the early stage of infection, since the replication mechanism of the diseased cells has not been effectively expressed, the replicated cells will mature and then die. As the virus continues to infect, it begins to encode E6 and E7 proteins, and E6 degrades the tumor suppressor gene TP53 through the ubiquitin degradation pathway. This blocks the normal growth cycle of cells and prevents cell maturation and apoptosis [(13); Figure 2]. E7 promotes DNA synthesis by interacting with retinoblastoma proteins (14). However, no significant increase in the number of E6 and E7 was observed when the apparent and integrated copies of the viral genome appeared in the same cell (15).

Normal cells transform into cancer cells, due to regulation of the cell cycle by HPV, or due to immune evasion behavior of HPV, which renders the virus undetectable for a long time (16). E6 reduces the expression of HPV antigen by reducing the expression of Cdh-1 on the surface of epithelial cells, whereas E7 prevents TAP1 from activating the immune mechanism of specific cytotoxic T lymphocytes (17). Moreover, E6 and E7 inhibit the transcription of Toll-like receptor 9 and reduce their ability to activate antigen-presenting cells. They also inhibit the synthesis of interferon (18, 19). Finally, the accumulation of genetic alterations and immune evasion causes HPV-induced intraepithelial neoplasia as well as transformation into cancer cells.

\section{HPV Vaccines}

\section{Immunogenicity of HPV Vaccines}

The cervical location of infection lacks secondary lymphoid tissues. Many memory B cells can reside, prepare antibodies to neutralize the virus (20). However, the levels of antibodies produced by natural infections are not enough to prevent reinfection. The HPV vaccine is immunized by inducing neutralizing antibodies (nAbs) with inactivated or attenuated natural pathogens (21).

The discovery of the pathogenic mechanism of HPV has led to the exploration of its prevention. The prophylactic HPV vaccine is the first one that has been clinically proven to prevent cancer, with landmark significance. In the 1990s, Romanowski et al. found virus-like particles (VLPs) were similar to natural virus particles and did not have the oncovirus genome (22). Studies have shown that in the absence of adjuvants, vaccination with L1VLP vaccines can induce high and long-lasting $\mathrm{nAb}$ titers to protect the host from attack by experimental viruses (23). However, the protective effect of L1 VLP on the host is limited by type. To achieve extensive protection, the HPV vaccine needs to contain several key types of L1VLP. The first vaccines were focus on HPV-16 and HPV-18 because of their carcinogenicity. These antibodies are thought to block HPV before entering the basal cell layer that proliferates through the epithelial surface by micro-scratch (24). Once, these antibodies meet HPV, they bind to the virus and prevent it from producing variant cells.

Several VLP-based vaccines have been approved by many countries around the world. 2vHPV was licensed in 2007 for use and consists of recombinant VLPs from L1 protein of the viral capsid of both HPV types 16 and 18 formulated in AS04. 4vHPV is composed of recombinant VLPs self-assembled from major capsid protein L1 of HPV types 6, 11, 16, 18 adjuvanted with neutral salt aluminum hydroxy phosphate sulfate (25). Merck has increased the 5 types VLP in their vaccine. Compared with 


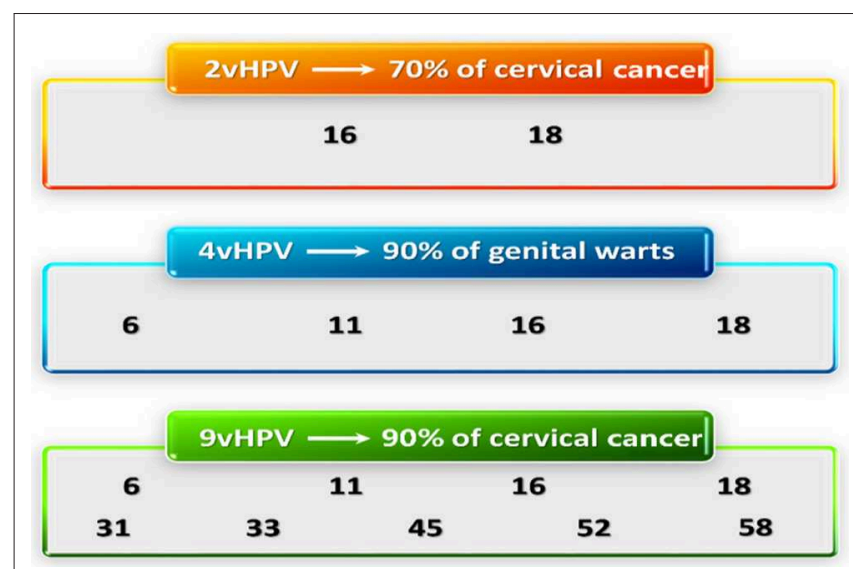

FIGURE 3 | HPV VLP types in VLP vaccines. VLPS in the 2VHPV, 4VHPV, and the 9vHPV are shown with the proportion of neoplastic disease attributed to each group. HPV, human papillomavirus; VLP, virus-like particle.
4vHPV, 9vHPV contains more HPV types, and L1 antigen load and adjuvant [(26); Figure 3].

Three vaccines have different immune components (Table 1). $2 \mathrm{vHPV}$ and $4 \mathrm{vHPV}$ can stimulate the immune system to produce enough HPV16 and HPV18 antibodies. 2vHPV vaccine can promote immunoglobulin $\mathrm{G}$ serum response, which is beneficial to the production of $\mathrm{T}$ helper cell 1 biased cell response $(27,28)$, whereas $4 \mathrm{vHPV}$ vaccine can produce a T helper cell 2 biased cell response that promotes immunoglobulin $\mathrm{G}$ and immunoglobulin A responses (29). Notable, a double-blind randomized controlled trial on 9vHPV reported that among women with HPV infection who have not completed three doses, the vaccine is more than $96 \%$ effective against persistent infections caused by HPV 31, $33,45,52$, and 58 for more than 6 months. After receiving three doses of 9vHPV 5 years, $\sim 77.5-100 \%$ women remained seropositive to the types (30). By comparing antibody titers of $9 \mathrm{vHPV}$ and $4 \mathrm{vHPV}$, similar antibody responses were observed in the two groups. There was no difference in the incidence of HPV6, HPV-11, HPV-16, and HPV-18-related diseases among the two groups, indicating the efficacy of 9vHPV for these types was non-inferior to the 4vHPV. Women treated with 9vHPV serum switched to HPV31, 33, 45, 52, and 58 in the seventh month, and the corresponding antibody titers were significantly higher than $4 \mathrm{vHPV}$ recipients. When this group was revaccinated again with one dose of 9vHPV, the antibody responses were higher at the first week and the first month after the fourth dose. This indicates that after three doses of the primary series of vaccines, the immune memory of all nine HPV types was affected (7). Compared with $4 \mathrm{vHPV}$ and 9vHPV vaccine, the $2 \mathrm{vHPV}$ vaccine has higher immunogenicity that elicits more HPV16/18 antibodies and stronger CD4+ T cell responses. Although the $2 \mathrm{vHPV}$ vaccine has the lowest antigen concentration among the three vaccines, it contains a late adjuvant AS04 that enhances immunogenicity. It directly persistent antibody responses, stimulates antigenpresenting cells, and elicits significant immune responses (31). Therefore, the $2 \mathrm{vHPV}$ vaccine can cause a more intense antibody neutralization reaction. Following vaccination, neutralizing antibodies transude from serum into cervicovaginal secretions where they may provide first-line defense against HPV (32).

The production cost of GlaxoSmithKline is not only more expensive but also more complicated than other system. Other vaccine manufacturers are trying to explore other alternatives (Table 2). It is notable that generating a suitable VLP can reduce the cost of production and the purified cation scheme induces virus particle neutralizing antibodies (33). There are some other HPV vaccines (Table 2). Several other companies are developing L1-based HPV vaccines not only in China but also in India, like Walvax (HPV-16 and HPV-18), China National Biotech Group (HPV-16, HPV-18, HPV-52, and HPV-58) and Health Guard (HPV16, 18, 58), Serum Institute of India (HPV6, 11, 16, 18), etc. (34). For example, Cecolin and Gecolin were developed by purifying L1 VLP from E. coli. Adeno associated virus (AAV) (35), bacteriophages (36), or those on a thioredoxin scaffold (37) can improve the immunogenicity of L2 protective epitopes (Table 2).

\section{Cross-Protection of HPV Vaccines}

Vaccine-mediated protection is not type-specific, and crossprotection exists after vaccination against a closely related type of the same species. Although $2 \mathrm{vHPV}$ is only related to HPV16 and HPV-18, it has shown 93\% effectiveness in preventing CIN3, regardless of HPV type $(38,39)$. Although the titer of cross-neutralizing antibodies is much lower than that of type-specific antibodies. The latest data confirms that crossprotection can be achieved by reducing the incidence of HPV 31, 33, and 45 exposure within 5 years after receiving the 2 vHPV vaccine $(40,41)$. This may be related to the unique adjuvant system. AS04 can induce inflammatory factors and $\mathrm{T}$ cells responses, and inhibit viral transcription or translation (34). These characteristics can be enhanced by exposure to nonvaccine HPV types. It also contributes to cross-protection of humoral and cellular prevention of HPV infection.

Further, 2vHPV can produce antibodies related to HPV$31,-33,-35,-45,-52$, and -58 through cross-protection, namely HPV $\alpha-7$ (related to HPV-18) or $\alpha-9$ (related to HPV-16) species (42). Regarding the durability of cross-reactions, studies have shown that cross-protection of $2 \mathrm{vHPV}$ lasts up to 8 years after vaccination, and there is no evidence that cross-protection of $2 \mathrm{vHPV}$ is weakened for women who have received three doses, which may be related to the genomic distance to HPV-16 or-18 (31). Although a single dose of $2 \mathrm{vHPV}$ or $4 \mathrm{vHPV}$ vaccine can obtain durable type-specific immunity. However, a study also showed that there was no cross-protection after a single $2 \mathrm{vHPV}$ or $4 \mathrm{vHPV}$ vaccine (43). The 9vHPV vaccine responded to HPV 6, 11, 16 and 18, and was as effective as the $4 \mathrm{vHPV}$ vaccine. The $9 \mathrm{vHPV}$ only has activity against HPV $6 / 11 / 16 / 18 / 31 / 33 / 45 / 52 / 58$, it does not prevent diseases caused by other types HPV (44).

\section{Adverse Reactions and Safety of HPV Vaccines}

Concerns about vaccine safety in some European countries have led to a decline in the acceptance of vaccines. The safety of vaccines has been controversial and has become one of 


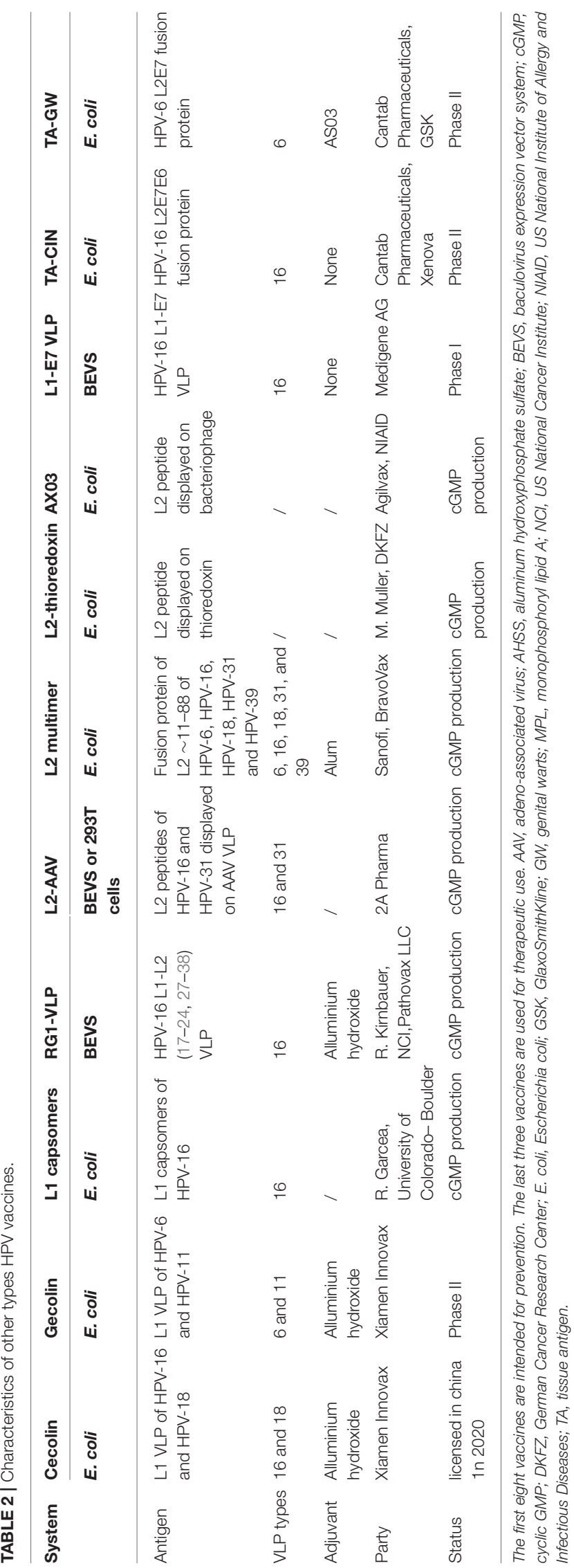

the main obstacles to public vaccination. Vaccination is not completely risk free, and it can also cause short term adverse reactions (AEs). The most common AE found currently are local injection site symptoms, including fatigue, headache, and muscle pain. Compared to $4 \mathrm{vHPV}$ vaccine, injection site reactions are more likely to occur in $2 \mathrm{vHPV}$ and $9 \mathrm{vHPV}$ vaccine $(45,46)$. Studies have found that in a post-marketing survey conducted on over 1,000 girls vaccinated with 2vHPV, myalgia was the most common systemic event, followed by fatigue and headache. Systemic AEs including fever, nausea, and dizziness have also been frequently reported (47). In 2013, the Japanese government terminated the HPV vaccination program due to the adverse effects of local pain syndrome events and instructed local health authorities not to promote the use of the vaccine until the end of the investigation of its side effects (44). Therefore, the vaccination rate in Japan fell sharply to below 3.9\%. The corresponding mortality rate of cervical cancer in women has also risen sharply $(46,48,49)$. Some countries also believe that HPV vaccination is associated with the occurrence of orthostatic tachycardia syndrome, whose causes are mostly unclear and difficult to determine (50). An assessment of vaccine safety in Denmark and Sweden, by analyzing 696,000 women who received the $4 \mathrm{vHPV}$ vaccine, did not find consistent evidence supporting the causal relationship between vaccines and autoimmune diseases. A study from France showed that vaccination with HPV did not increase the risk of autoimmune outcomes (51). Another analysis of national data in these countries involved 4 million women, among the 800,000 women who received the $4 \mathrm{vHPV}$ vaccine, there was no increase in the incidence of multiple sclerosis or demyelinating diseases after vaccination. In Finland, largescale statistics on vaccinated adolescents have not been found to increase the incidence of autoimmune diseases (52).

Over 15 years of active monitoring and investigation of any safety signals detected by the HPV vaccine, from pre-licensing active and passive monitoring were conducted. Therefore, the $\mathrm{HPV}$ vaccine is one of the most well-studied vaccines to date. The Global Vaccine Safety Advisory Committee has been studying safety-related issues of HPV vaccination and has publicly announced the continued safety and benefits of vaccines seven times (53). Moreover, safety data on vaccination from six Asian countries (10 studies, eight on 2vHPV vaccine, and two on $4 \mathrm{vHPV}$ vaccine) showed that the HPV vaccine was also safe for Asian populations (54). Many experimental data show that the vaccine does not cause any serious or unexpected adverse events. Therefore, we should restore public confidence in the safety of HPV vaccination by expanding the scope of vaccination and creating awareness about its benefits and safety. This will play a decisive role in the HPV vaccination program and consequently the risk of HPV-associated cancers.

Pregnancy tests were not required before vaccination, and no adverse outcomes occurred among vaccinated pregnant women. However, HPV vaccination during pregnancy is still not recommended. If pregnancy is confirmed after vaccination, the remaining dose should be postponed to the end of pregnancy (41, 55). There are also some reactions during vaccination, although these reactions are caused by the vaccination process, and not by the vaccine itself (56). 


\section{Immunization Procedure of HPV Vaccines}

In the US, the cost of receiving a three-dose $2 \mathrm{vHPV}$ or $4 \mathrm{vHPV}$ vaccine is between $\$ 350$ and $\$ 500(57,58)$. Reduction of the economic cost of vaccines is particularly important. Research shows that the two-dose of $2 \mathrm{vHPV}$ and $4 \mathrm{vHPV}$ vaccine injections are not inferior to the three-dose regimen for adolescents (58). A study was initiated in India to research whether less than three doses of vaccine in 6 month could be non-inferior to three doses for girls aged 10-18 years (59).The study shows that the immunogenicity after two doses of HPV vaccination was as effective as three doses. Therefore, two doses are non-inferior to three doses in preventing HPV related diseases in girls aged 15-18 years (59). WHO has proposed two doses of the $2 \mathrm{vHPV}$ and $4 \mathrm{vHPV}$ vaccines for people under the age of 15 and three for women over the age of 15 . However, the two dose effects are very close to that of three doses provided the two doses of the antibody are separated by at least 6 months (58). In recent years, in some countries, the three-dose immunization program has been promoted and licensed to a two-dose immunization program, and two doses of $2 \mathrm{vHPV}$ and $4 \mathrm{vHPV}$ vaccines have been given to adolescents (9-13 years old or 14 years old) (60). This problem is particularly prominent because of the high cost of the multi-dose regimen and the cumbersome vaccination process, this issue is particularly important. The second dose can be used flexibly, receiving a two-dose instead of a threedose of the HPV vaccine may help to considerably reduce costs, thereby increasing vaccination coverage. This will potentially protect more girls from cervical cancer in low-income countries (61). An increasing number of trials are trying to demonstrate the effect of one dose of vaccine. In a large case-control from India, women who received a single dose of $4 \mathrm{vHPV}$ vaccine were followed for up to 7 years and the result indicates that this vaccine can provide lasting protection against HPV16 and HPV18 related diseases (62). Although inferior to two or three doses, single dose is immunogenic to HPV16 and HPV18 (59). Single-dose vaccination is more affordable, will simplify the logistics of vaccine delivery, save significant vaccination costs, and is particularly beneficial for wider implementation in low and middle-income countries. However, research into the efficacy and immune response of single-dose HPV vaccines may need to be expanded to other target groups, such as boys, alternative age groups, and HIV-positive individuals. All licensed HPV vaccines should be evaluated, and those currently being developed (34). Data about efficiency of a single dose of vaccine are only up to 7 years old. Therefore, the protection provided by single-dose vaccines still requires long-term assessment.

Since April 2016, the European Medicines Agency has approved a two-dose regimen for 9vHPV for teenagers between the ages of 9 and 14, and those who started using the 9vHPV vaccine after the age of 15 or people with immunodeficiency diseases nevertheless recommend a three-dosage regimen (47).

For these three vaccines, people who have received the first vaccine are advised to use the same one to complete the immunization process $(63,64)$. With the prevalence of the $9 \mathrm{vHPV}$ vaccine, there may be uncertainty about completion of the immunization process starting with one $2 \mathrm{vHPV}$ or $4 \mathrm{vHPV}$ vaccines, and people who have completed immunization with a
2 vHPV or 4 vHPV vaccine whether need to revaccinate (65). For women who have not completed the three dose immunization of $2 \mathrm{vHPV}$ or $4 \mathrm{vHPV}$ vaccines (66) need an additional injection of $9 \mathrm{vHPV}$ between 6 and 12 months after the second dose of $2 \mathrm{vHPV}$ or $4 \mathrm{vHPV}$ vaccines, to completely prevent HPV related diseases. However, in order to obtain some additional protection for other types, should be revaccinated with 9vHPV vaccine after 6-12 months, no evidence supports that one single dose vaccine will protect comprehensively (65). For women who have completed three doses of $2 \mathrm{vHPV}$ or $4 \mathrm{vHPV}$ vaccine, they can receive two doses of 9vHPV vaccine in 6-12 months (67). A randomized trial showed that three doses of a $9 \mathrm{vHPV}$ vaccine were given to a girl who had already received the $4 \mathrm{vHPV}$ vaccine previously, the HPV 16, 18 titers was two to three times higher than another girl who only receiving 9vHPV vaccine. However, the antiHPV 31/33/45/52/58 titers were significantly reduced. Moreover, the resulting anti-HPV 31/33/45/52/58 titers are not likely to provide HPV-specific infection protection (68). Given these results and economic cost-effectiveness, it is recommended that a 9vHPV vaccine be used to complete an incomplete vaccination program to expand the scope of protection. For women who have completed three doses of $2 \mathrm{vHPV}$ or $4 \mathrm{vHPV}$, it is recommended that a full injection of the 9vHPV 1 year later be completed.

\section{Progress in Vaccination of HPV Vaccines}

As a public health issue, cervical cancer can be eliminated by boosting HPV vaccination, cervical cancer screening and related treatments. WHO called for global action to reach this goal in May 2018 (69). A systematic review of the clinical experience of HPV vaccines after use has revealed that its impact in the world is becoming more pronounced, particularly in countries with high vaccination rates. In such countries, the infection of HPV $6 / 11 / 16 / 18$ was reduced by more than $90 \%$, the incidence of genital warts caused by HPV decreased by $90 \%$, and the low-grade lesions of the cervix were reduced by $45 \%$ (Figure 4). For other sites, published data shows a significant impact of the 9vHPV vaccine on preventing HPV related diseases worldwide (1). However, the process of vaccine introduction has been relatively slow due to the complex production process, excessive costs, the production volume of vaccines cannot meet the demand, the understanding of vaccines is insufficient and the attitude toward vaccination (70).

The predominant factor making vaccines failing to achieve the desired results is insufficient population coverage (71). However, in most highly developed countries, vaccine coverage has not reached effective levels. Crucially, vaccine coverage is $<1 \%$ in many underdeveloped areas. By October 2014, the vaccination rate was $53.4 \%$ in North America, 36.4\% in Europe, $41.1 \%$ in Oceania, $22.1 \%$ in South America, and only 1-2\% of $10-20$ year old women in Africa and Asia [(70); Figure 4]. Reducing the cost of vaccines (including the need for refrigeration of the vaccine), effective programs and measures adopted by governments are the key factors for long-term success (72). Simultaneously, screening at least one type of cervical cancer for women over 30 years old is an effective way to prevent cervical cancer, although it will increase the government's financial burden. Adolescent women before the sexual debut are considered the best targets for 


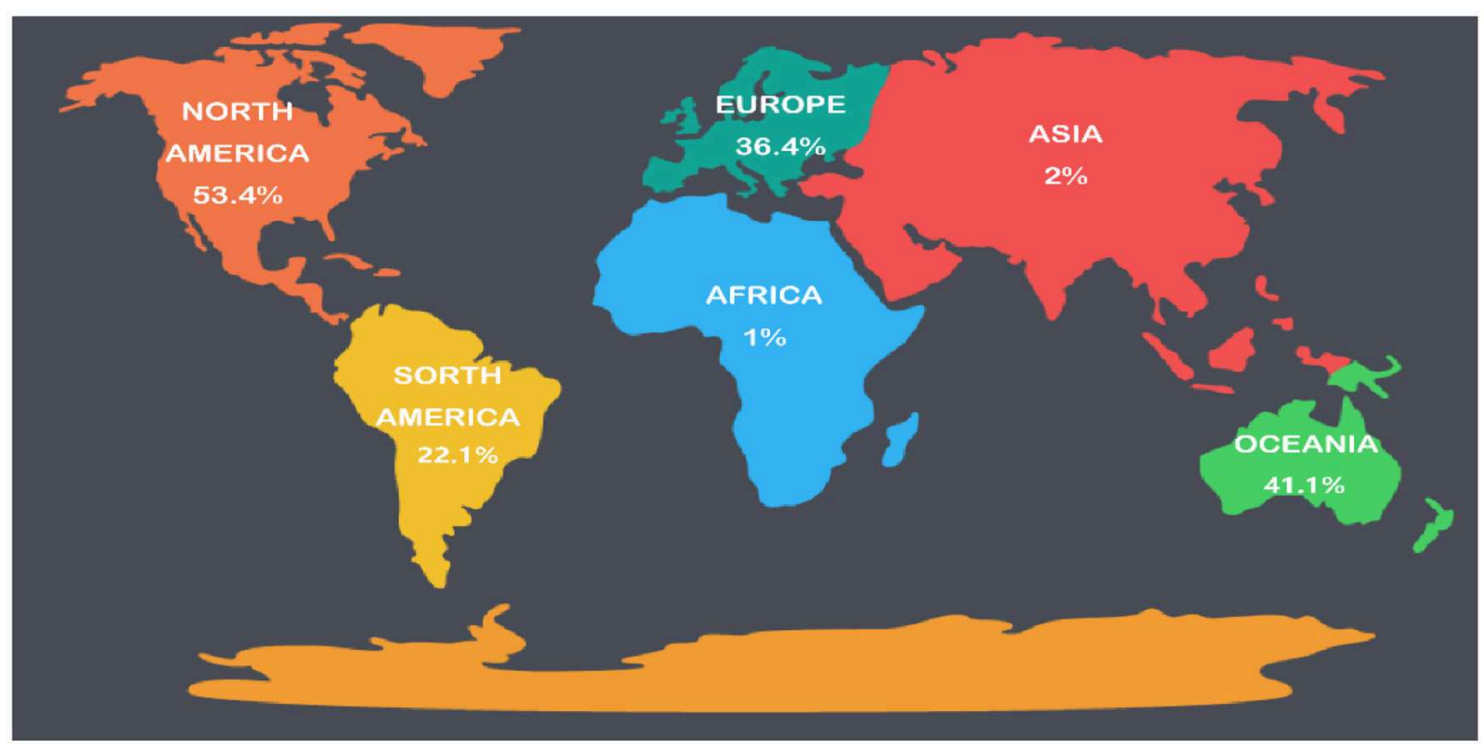

FIGURE 4 | Worldwide HPV vaccination rates. HPV, human papillomavirus.

cervical cancer vaccination (41). However, given the long-lasting response to vaccination, it is likely that a children's program will be implemented, such as placing the HPV vaccine in the same bottle as other children's vaccines given under the existing program, to reduce the need for infrastructure. After vaccination of elderly women, the effect is poor, and it has less effect on reducing the incidence of cervical cancer (34). Therefore, vaccination programs targeting specific populations will require further investigation. Furthermore, cost-effective models are still needed to determine the age range and screening interval for $\mathrm{HPV}$ vaccination in public health programs (73).

\section{Progress in Vaccination of HPV Vaccines in Developed Countries}

Since women are the high-risk group of HPV-related diseases, the focus of vaccination programs has always been on women, although in some developed areas vaccination of adolescent men is recommended. Across Europe, HPV vaccination has been implemented in all 28 European Union countries, with vaccination rates as low as $10 \%$ in Poland (74) and $43 \%$ in Luxembourg (25). The vaccination rate in Italy is $27-83 \%(26)$, $86 \%$ in the United Kingdom (75), in Belgium Flanders as high as $90 \%$ (76). Australia is one of the best to complete the vaccination program and the high efficiency of the 4vHPV vaccine has reduced the infection rate of HPV-6, HPV-11, HPV-16, and HPV-18 in women aged 25 years or older. In male subjects, the prevalence and morbidity of HPV in unvaccinated men was reduced by $70 \%$, and these data suggest that even unvaccinated women can obtain high coverage from vaccines. Australia has changed its cervical cancer screening program to 5 years in 2017 (77). If Australia maintains current HPV vaccination and screening coverage, the annual new cases of cervical cancer may be reduced to $<6$ cases per 100,000 women by 2020 , and to 4 per 100,000 women by 2028 (78). Hence, as a public health issue, Australia might be the first country to eliminate cervical cancer in 20 years (79). A similar situation exists in the Danish survey (80). After the implementation of HPV vaccination program in Britain in 2008, the HPV infection rate showed a decreased trend. Investigations from Scotland also suggest a notable drop in HPV infection and cervical lesions. By analyzing 8854 samples of HPV genotyping, the infection rate of HPV 16/18 decreased from 30.0 to $4.5 \%$. The effective rate of $2 \mathrm{vHPV}$ vaccine in women aged $12-13$ was $89.1 \%$, and it was confirmed that the HPV type was highly cross-protected in the $2 \mathrm{vHPV}$ vaccine test (41).

\section{Progress in Vaccination of HPV Vaccines in China}

In China, cervical cancer is an important social health issue: there are about 140,000 added cases and more than 34,000 deaths every year (81). This is consistent with trends in sociocultural norms related to sexual behavior, particularly in young people. The incidence of HPV-related diseases in China increased significantly since 2000, particularly women in their twenties. The trend is largely attributable to lifestyle changes, such as changing sexual behavior (82). The annual incidence of cervical cancer in Chinese urban and rural areas has increased by 9.8 and 15.5\%, respectively (83). Therefore, early HPV vaccination in the population can greatly reduce the risk of cervical cancer. Furthermore, it is effective to introduce HPV vaccination timeously for public health in China. The China Food and Drug Administration approved the 2vHPV vaccine in July 2016, which was a groundbreaking decision by the Chinese authorities. Long-term follow-up of clinical studies of the efficacy of $2 \mathrm{vHPV}$ vaccine in Chinese women has shown that the effect of the vaccine is consistent with the results of a global study (84). The vaccine is highly immunogenic to 
Chinese women and does not cause serious adverse reactions. It is effective for HPV 16, 18-related lesions, haptic infections and persistent infections of HPV 31/33/45. In the years following the first injection, the anti-HPV 16/18 antibody response is high and persistent, which is expected to reduce the risk of HPV 16/18 infection in Chinese women. An evaluation of the efficacy and safety of a Chinese woman's $4 \mathrm{HPV}$ vaccine for $20-45$ years of age indicates that the $4 \mathrm{vHPV}$ vaccine has a continuing effect on anal infections and diseases (including highgrade cervical disease) in Chinese women. The time is up to 6.5 years (85). In China, HPV 16/18/58/52/33 are most commonly associated with cervical lesions, suggesting that women infected with HPV 58/52/33 account for a higher proportion of Chinese population than other countries (86). A Chinese bivalent HPV vaccine, including HPV type 16 and 18 VLPs, has been licensed in China in 2020 (Table 1) and completed phase 2-3 trials in terms of efficacy and safety (34). Is has been licensed in China due to the effectiveness and affordability of Chinese HPV vaccine, and the coverage of HPV vaccination in China will increase significantly.

\section{CONCLUSION}

Public surveillance can promote the effectiveness and safety of HPV vaccines. Furthermore, there are many ways to obtain vaccination information (such as the media) and the information has been misled by misinformation, speculation, and suspicion. This has led to public distrust of the pharmaceutical industry and biomedical technology $(87,88)$. Moreover, parents and doctors also doubt vaccine safety and have negative attitudes toward it. In addition, parents are concerned that vaccination of HPV vaccine will be examined as acquiescence in sexual activity. The first step to improve the completion rate of HPV vaccine doses for women and men is the training of pediatrics, general practitioners and obstetricians and gynecologists (89). Therefore, we should strive to disseminate vaccine-related science, policies, and recommendations to the non-professional public through various media forms. This will reduce the incidence of cervical cancer in a substantive way (89).

\section{REFERENCES}

1. de Sanjose S, Serrano B, Tous S, Alejo M, Lloveras B, Quiros B, et al. Burden of human papillomavirus (HPV)-related cancers attributable to HPVs 6/11/16/18/31/33/45/52 and 58. JNCI Cancer Spectr. (2018) 2:pky045. doi: 10.1093/jncics/pky045

2. Zur H. Cancers in humans: a lifelong search for contributions of infectious agents, autobiographic notes. Ann Rev Virol. (2019) 6:128. doi: 10.1146/annurev-virology-092818-015907

3. Markowitz LE, Tsu V, Deeks SL, Cubie H, Wang SA, Vicari AS, et al. Human papillomavirus vaccine introduction-the first five years. Vaccine. (2012) :F139-48. doi: 10.1016/j.vaccine.2012.05.039

4. Bogani G, Leone Roberti Maggiore U, Signorelli M, Martinelli F, Ditto A, Sabatucci I, et al. The role of human papillomavirus vaccines in cervical cancer: prevention and treatment. Crit Rev Oncol Hematol. (2018) 122:927. doi: 10.1016/j.critrevonc.2017.12.017
Deaths from oropharyngeal cancer (HPV-related cancers) are projected to increase in 2020, particularly in developed countries (90). Therefore, it is necessary to work toward the full implementation of the vaccination program globally, both for men and women, and to extend the coverage to all medically significant genotypes. In near future, we should also conduct further research on the vaccine itself, optimization of the age of vaccination and the most effective dosage schedule. An increasing number of HPV vaccines are undergoing clinical trials or licensing, such as the Chinese bivalent vaccine, the quadrivalent HPV vaccine in India, and HPV type 6, 11, 16, and 18 VLPs produced by the Indian Serum Institute (Pune, India) (59). Canada has started pairing 9vHPV and 2vHPV (91). There are also studies comparing the effect of one dose of $2 \mathrm{vHPV}$ or 9vHPV vaccine with two doses of vaccine (90). These methods can reduce the cost of vaccination and induce a stronger immune response. In conclusion, the latest progress of the HPV vaccine in the immunogenicity, safety, efficacy, latest vaccination concepts, and strategies were reviewed and the prospect solutions were discussed. There are still many challenges in the widespread vaccination of the HPV vaccine. This review provides a theoretical base and applications for the treatment of HPV-related diseases and the further application of the HPV vaccine.

\section{AUTHOR CONTRIBUTIONS}

$\mathrm{XZ}, \mathrm{YW}$, and GL were involved in the study conception and design. LS and XY participated in data acquisition, analysis, and interpretation. XZ and YL wrote the manuscript. All authors have read and approved the manuscript for publication.

\section{FUNDING}

This study was supported by Natural Science Foundation of China (81802613, 81772757, and 81901880), Jilin Science and Technology Agency funding (20180101114JC and 20200201422JC), Finance Department of Jilin Province (2019SCZT016 and 3D518V623429), and Education Department of Jilin Province (JJKH20201080KJ).

5. Khan MJ, Castle PE, Lorincz AT, Wacholder S, Sherman M, Scott DR, et al. The elevated 10-year risk of cervical precancer and cancer in women with human papillomavirus (HPV) type 16 or 18 and the possible utility of type-specific HPV testing in clinical practice. J Natl Cancer Inst. (2005) 97:1072-9. doi: 10.1093/jnci/dji187

6. Winer RL, Feng Q, Hughes JP, O’Reilly S, Kiviat NB, Koutsky LA. Risk of female human papillomavirus acquisition associated with first male sex partner. J Infect Dis. (2008) 197:279-82. doi: 10.1086/ 524875

7. Toh ZQ, Kosasih J, Russell FM, Garland SM, Mulholland EK, Licciardi PV. Recombinant human papillomavirus nonavalent vaccine in the prevention of cancers caused by human papillomavirus. Infect Drug Resist. (2019) 12:195167. doi: 10.2147/IDR.S178381

8. Crosbie EJ, Einstein MH, Franceschi S, Kitchener HC. Human papillomavirus and cervical cancer. Lancet. (2013) 382:88999. doi: 10.1016/S0140-6736(13)60022-7 
9. Bouvard V, Baan R, Straif K, Grosse Y, Secretan B, El Ghissassi F, et al. A review of human carcinogens-Part B: biological agents. Lancet Oncol. (2009) 10:321-2. doi: 10.1016/S1470-2045(09)70096-8

10. Soudeyns C, Speybroeck N, Brisson M, Mossong J, Latsuzbaia A. HPV vaccination and sexual behaviour in healthcare seeking young women in luxembourg. Peer J. (2020) 8:e8516. doi: 10.7717/peerj.8516

11. Harper DM, DeMars LR. Corrigendum to "HPV vaccines-a review of the first decade”. Gynecol Oncol. (2017) 147:489. doi: 10.1016/j.ygyno.2017.08.019

12. Doorbar J. The papillomavirus life cycle. J Clin Virol. (2005) 32(Suppl 1):S715. doi: 10.1016/j.jcv.2004.12.006

13. Tommasino M, Accardi R, Caldeira S, Dong W, Malanchi I, Smet A, et al. The role of TP53 in Cervical carcinogenesis. Hum Mutat. (2003) 21:30712. doi: 10.1002/humu. 10178

14. Einstein MH, Leanza S, Chiu LG, Schlecht NF, Goldberg GL, Steinberg BM, et al. Genetic variants in TAP are associated with high-grade cervical neoplasia. Clin Cancer Res. (2009) 15:1019-23. doi: 10.1158/1078-0432.CCR-08-1207

15. Fan Y, Shen Z. The clinical value of HPV E6/E7 and STAT3 mRNA detection in cervical cancer screening. Pathol Res Prac. (2018) 214:76775. doi: 10.1016/j.prp.2018.02.003

16. Piersma SJ. Immunosuppressive tumor microenvironment in cervical cancer patients. Cancer Microenviron. (2011) 4:36175. doi: 10.1007/s12307-011-0066-7

17. Hasan UA, Bates E, Takeshita F, Biliato A, Accardi R, Bouvard V, et al. TLR9 expression and function is abolished by the cervical cancerassociated human papillomavirus type 16. J Immunol. (2007) 178:318697. doi: 10.4049/jimmunol.178.5.3186

18. Park JS, Kim EJ, Kwon HJ, Hwang ES, Namkoong SE, Um SJ. Inactivation of interferon regulatory factor-1 tumor suppressor protein by HPV E7 oncoprotein. Implication for the E7-mediated immune evasion mechanism in cervical carcinogenesis. J Biol Chem. (2000) 275:6764-9. doi: 10.1074/jbc.275.10.6764

19. Ronco LV, Karpova AY, Vidal M, Howley PM. Human papillomavirus 16 E6 oncoprotein binds to interferon regulatory factor-3 and inhibits its transcriptional activity. Genes Dev. (1998) 12:206172. doi: 10.1101/gad.12.13.2061

20. Schwarz TF, Kocken M, Petaja T, Einstein MH, Spaczynski M, Louwers JA, et al. Correlation between levels of human papillomavirus (HPV)-16 and 18 antibodies in serum and cervicovaginal secretions in girls and women vaccinated with the HPV-16/18 AS04-adjuvanted vaccine. Hum Vaccin. (2010) 6:1054-61. doi: 10.4161/hv.6.12.13399

21. Geraets D, Alemany L, Guimera N, de Sanjose S, de Koning M, Molijn A, et al. Detection of rare and possibly carcinogenic human papillomavirus genotypes as single infections in invasive cervical cancer. J Pathol. (2012) 228:534-43. doi: 10.1002/path.4065

22. Romanowski B, Schwarz TF, Ferguson LM, Ferguson M, Peters K, Dionne $\mathrm{M}$, et al. Immune response to the HPV-16/18 AS04-adjuvanted vaccine administered as a 2 -dose or 3 -dose schedule up to 4 years after vaccination: results from a randomized study. Hum Vaccin Immunother. (2014) 10:115565. doi: $10.4161 /$ hv. 28022

23. Suzich JA, Ghim SJ, Palmer-Hill FJ, White WI, Tamura JK, Bell JA, et al. Systemic immunization with papillomavirus L1 protein completely prevents the development of viral mucosal papillomas. Proc Natl Acad Sci USA. (1995) 92:11553-7. doi: 10.1073/pnas.92.25.11553

24. Roberts JN, Buck CB, Thompson CD, Kines R, Bernardo M, Choyke PL, et al. Genital transmission of HPV in a mouse model is potentiated by nonoxynol-9 and inhibited by carrageenan. Nat Med. (2007) 13:85761. doi: $10.1038 / \mathrm{nm} 1598$

25. Latsuzbaia A, Arbyn M, Weyers S, Mossong J. Human papillomavirus vaccination coverage in Luxembourg - implications of lowering and restricting target age groups. Vaccine. (2018) 36:2411-6. doi: 10.1016/j.vaccine.2018.03.054

26. Lee LY, Garland SM. Human papillomavirus vaccination: the population impact. F1000Res. (2017) 6:866. doi: 10.12688/f1000research.10691.1

27. Scherpenisse M, Schepp RM, Mollers M, Meijer CJ, Berbers GA, van der Klis FR. Characteristics of HPV-specific antibody responses induced by infection and vaccination: cross-reactivity, neutralizing activity, avidity and $\operatorname{IgG}$ subclasses. PLoS ONE. (2013) 8:e74797. doi: 10.1371/journal.pone.0074797
28. Herrin DM, Coates EE, Costner PJ, Kemp TJ, Nason MC, Saharia KK, et al. Comparison of adaptive and innate immune responses induced by licensed vaccines for human papillomavirus. Hum Vaccin Immunother. (2014) 10:3446-54. doi: 10.4161/hv.34408

29. Ruiz W, McClements WL, Jansen KU, Esser MT. Kinetics and isotype profile of antibody responses in rhesus macaques induced following vaccination with HPV 6, 11, 16 and 18 L1-virus-like particles formulated with or without Merck aluminum adjuvant. J Immune Based Ther Vaccines. (2005) 3:2. doi: 10.1186/1476-8518-3-2

30. Huh WK, Joura EA, Giuliano AR, Iversen OE, de Andrade RP, Ault KA, et al. Final efficacy, immunogenicity, and safety analyses of a nine-valent human papillomavirus vaccine in women aged 16-26 years: a randomised, doubleblind trial. Lancet. (2017) 390:2143-59. doi: 10.1016/S0140-6736(17)31821-4

31. Bogaards JA, van der Weele P, Woestenberg PJ, van Benthem BHB, King AJ. Bivalent human papillomavirus (HPV) vaccine effectiveness correlates with phylogenetic distance from HPV vaccine types 16 and 18. J Infect Dis. (2019) 220:1141-6. doi: 10.1093/infdis/jiz280

32. Schwarz TF, Leo O. Immune response to human papillomavirus after prophylactic vaccination with AS04-adjuvanted HPV-16/18 vaccine: improving upon nature. Gynecol Oncol. (2008) 110(3 Suppl. 1):S1-10. doi: 10.1016/j.ygyno.2008.05.036

33. Schiller JT, Müller M. Next generation prophylactic human papillomavirus vaccines. Lancet Oncol. (2015) 16:e21725. doi: 10.1016/S1470-2045(14)71179-9

34. Roden RBS, Stern PL. Opportunities and challenges for human papillomavirus vaccination in cancer. Nat Rev Cancer. (2018) 18:240-54. doi: 10.1038/nrc.2018.13

35. Harkenrider MM, Martin B, Nieto K, Small C, Aref I, Bergman $\mathrm{D}$, et al. Development of AAVLP(HPV16/31L2) particles as broadly protective HPV vaccine candidate. PloS ONE. (2012) 7:e39741. doi: 10.1371/journal.pone.0039741

36. Tumban E, Muttil P, Escobar CA, Peabody J, Wafula D, Peabody DS, et al. Preclinical refinements of a broadly protective VLP-based HPV vaccine targeting the minor capsid protein, L2. Vaccine. (2015) 33:334653. doi: 10.1016/j.vaccine.2015.05.016

37. Rubio I, Bolchi A, Moretto N, Canali E, Gissmann L, Tommasino M, et al. Potent anti-HPV immune responses induced by tandem repeats of the HPV16 L2 (20 - 38) peptide displayed on bacterial thioredoxin. Vaccine. (2009) 27:1949-56. doi: 10.1016/j.vaccine.2009.01.102

38. Lehtinen $M$, Paavonen J, Wheeler CM, Jaisamrarn U, Garland SM, Castellsague X, et al. Overall efficacy of HPV-16/18 AS04-adjuvanted vaccine against grade 3 or greater cervical intraepithelial neoplasia: 4-year end-ofstudy analysis of the randomised, double-blind PATRICIA trial. Lancet Oncol. (2012) 13:89-99. doi: 10.1016/S1470-2045(11)70286-8

39. Lang Kuhs KA, Porras C, Schiller JT, Rodriguez AC, Schiffman M, Gonzalez P, et al. Effect of different human papillomavirus serological and DNA criteria on vaccine efficacy estimates. Am J Epidemiol. (2014) 180:599607. doi: 10.1093/aje/kwu168

40. Cameron RL, Kavanagh K, Pan J, Love J, Cuschieri K, Robertson C, et al. Human papillomavirus prevalence and herd immunity after introduction of vaccination program, Scotland, 2009-2013. Emerg Infect Dis. (2016) 22:5664. doi: 10.3201/eid2201.150736

41. Kavanagh K, Pollock KG, Cuschieri K, Palmer T, Cameron RL, Watt $\mathrm{C}$, et al. Changes in the prevalence of human papillomavirus following a national bivalent human papillomavirus vaccination programme in Scotland: a 7-year cross-sectional study. Lancet Infect Dis. (2017) 17:1293302. doi: 10.1016/S1473-3099(17)30468-1

42. de Villiers EM. Cross-roads in the classification of papillomaviruses. Virology. (2013) 445:2-10. doi: 10.1016/j.virol.2013.04.023

43. Cuschieri K, Kavanagh K, Moore C, Bhatia R, Love J, Pollock KG. Impact of partial bivalent HPV vaccination on vaccine-type infection: a population-based analysis. Br J Cancer. (2016) 114:1261-4. doi: 10.1038/bjc. 2016.97

44. Joura EA, Giuliano AR, Iversen OE, Bouchard C, Mao C, Mehlsen J, et al. A 9valent HPV vaccine against infection and intraepithelial neoplasia in women. N Engl J Med. (2015) 372:711-23. doi: 10.1056/NEJMoa1405044

45. Einstein MH, Baron M, Levin MJ, Chatterjee A, Edwards RP, Zepp F, et al. Comparison of the immunogenicity and safety of cervarix and gardasil human 
papillomavirus (HPV) cervical cancer vaccines in healthy women aged 18-45 years. Hum Vaccin. (2009) 5:705-19. doi: 10.4161/hv.5.10.9518

46. Vesikari T, Brodszki N, van Damme P, Diez-Domingo J, Icardi G, Petersen LK, et al. A randomized, double-blind, phase III study of the immunogenicity and safety of a 9-valent human papillomavirus L1 virus-like particle vaccine (V503) versus gardasil(R) in 9-15-year-old girls. Pediatr Infect Dis J. (2015) 34:992-8. doi: 10.1097/INF.0000000000000773

47. van Klooster TM, Kemmeren JM, van der Maas NA, de Melker HE. Reported adverse events in girls aged 13-16 years after vaccination with the human papillomavirus (HPV)-16/18 vaccine in the Netherlands. Vaccine. (2011) 29:4601-7. doi: 10.1016/j.vaccine.2011.04.050

48. Van Damme P, Olsson SE, Block S, Castellsague X, Gray GE, Herrera T, et al. Immunogenicity and safety of a 9-valent HPV vaccine. Pediatrics. (2015) 136:e28-39. doi: 10.1542/peds.2014-3745

49. Van Damme P, Meijer C, Kieninger D, Schuyleman A, Thomas S, Luxembourg A, et al. A phase III clinical study to compare the immunogenicity and safety of the 9-valent and quadrivalent HPV vaccines in men. Vaccine. (2016) 34:4205-12. doi: 10.1016/j.vaccine.2016.06.056

50. Ward D, Thorsen NM, Frisch M, Valentiner-Branth P, Molbak K, Hviid A. A cluster analysis of serious adverse event reports after human papillomavirus (hpv) vaccination in Danish girls and young women, September 2009 to August 2017. Euro Surveill. (2019) 24(19). doi: 10.2807/1560-7917.ES.2019.24.19.1800380

51. Giannini SL, Hanon E, Moris P, Van Mechelen M, Morel S, Dessy F, et al. Enhanced humoral and memory B cellular immunity using HPV16/18 L1 VLP vaccine formulated with the MPL/aluminium salt combination (AS04) compared to aluminium salt only. Vaccine. (2006) 24:5937-49. doi: 10.1016/j.vaccine.2006.06.005

52. Lehtinen M, Eriksson T, Apter D, Hokkanen M, Natunen K, Paavonen J, et al. Safety of the human papillomavirus (HPV)-16/18 AS04-adjuvanted vaccine in adolescents aged 12-15 years: interim analysis of a large communityrandomized controlled trial. Hum Vaccin Immunother. (2016) 12:317785. doi: 10.1080/21645515.2016.1183847

53. Angelo MG, David MP, Zima J, Baril L, Dubin G, Arellano F, et al. Pooled analysis of large and long-term safety data from the human papillomavirus16/18-AS04-adjuvanted vaccine clinical trial programme. Pharmacoepidemiol Drug Saf. (2014) 23:466-79. doi: 10.1002/pds.3554

54. Setiawan D, Luttjeboer J, Pouwels KB, Wilschut JC, Postma MJ. Immunogenicity and safety of human papillomavirus (HPV) vaccination in Asian populations from six countries: a meta-analysis. Jpn J Clin Oncol. (2017) 47:265-76. doi: 10.1093/jjco/hyw192

55. Goss MA, Lievano F, Buchanan KM, Seminack MM, Cunningham ML, Dana A. Final report on exposure during pregnancy from a pregnancy registry for quadrivalent human papillomavirus vaccine. Vaccine. (2015) 33:34228. doi: 10.1016/j.vaccine.2015.04.014

56. Centers for Disease Control Prevention. Syncope after vaccinationUnited States, January 2005-July 2007. MMWR Morb Mortal Wkly Rep. (2008) 57:457-60. doi: 10.1001/jama.299.21.2502

57. Tota JE, Jiang M, Ramanakumar AV, Walter SD, Kaufman JS, Coutlee F, et al. Epidemiologic evaluation of human papillomavirus type competition and the potential for type replacement post-vaccination. PLoS ONE. (2016) 11:e0166329. doi: 10.1371/journal.pone.0166329

58. Tota JE, Ramanakumar AV, Villa LL, Richardson H, Burchell AN, Coutlee F, et al. Cervical infection with vaccine-associated human papillomavirus (HPV) genotypes as a predictor of acquisition and clearance of other HPV infections. J Infect Dis. (2016) 214:676-84. doi: 10.1093/infdis/jiw215

59. Sankaranarayanan R, Basu P, Kaur P, Bhaskar R, Singh GB, Denzongpa $\mathrm{P}$, et al. Current status of human papillomavirus vaccination in India's cervical cancer prevention efforts. Lancet Oncol. (2019) 20:e637-e44. doi: 10.1016/S1470-2045(19)30531-5

60. Toh ZQ, Russell FM, Reyburn R, Fong J, Tuivaga E, Ratu T, et al. Sustained antibody responses 6 years following 1, 2, or 3 doses of quadrivalent human papillomavirus (HPV) vaccine in adolescent Fijian girls, and subsequent responses to a single dose of bivalent HPV vaccine: a prospective cohort study. Clin Infect Dis. (2017) 64:852-9. doi: 10.1093/ofid/ ofy147

61. Olsson SE, Kjaer SK, Sigurdsson K, Iversen OE, Hernandez-Avila M, Wheeler CM, et al. Evaluation of quadrivalent HPV 6/11/16/18 vaccine efficacy against cervical and anogenital disease in subjects with serological evidence of prior vaccine type HPV infection. Hum Vaccin. (2009) 5:696704. doi: $10.4161 /$ hv.5.10.9515

62. Sankaranarayanan R, Joshi S, Muwonge R, Esmy PO, Basu P, Prabhu P, et al. Can a single dose of human papillomavirus (HPV) vaccine prevent cervical cancer? Early findings from an Indian study. Vaccine. (2018) 36:478391. doi: 10.1016/j.vaccine.2018.02.087

63. Moreira ED Jr, Block SL, Ferris D, Giuliano AR, Iversen OE, Joura EA, et al. Safety profile of the 9-valent hpv vaccine: a combined analysis of 7 phase III clinical trials. Pediatrics. (2016) 138: e20154387. doi: 10.1542/peds.2015-4387

64. Bansal D, Bhagat A, Schifano F, Gudala K. Role of patient-reported outcomes and other efficacy endpoints in the drug approval process in Europe (2008-2012). J Epidemiol Glob Health. (2015) 5:385-95. doi: 10.1016/j.jegh.2015.04.006

65. Van Damme P, Bonanni P, Bosch FX, Joura E, Kjaer SK, Meijer CJ, et al. Use of the nonavalent HPV vaccine in individuals previously fully or partially vaccinated with bivalent or quadrivalent HPV vaccines. Vaccine. (2016) 34:757-61. doi: 10.1016/j.vaccine.2015.12.063

66. Human papillomavirus vaccines: WHO position paper, October 2014-Recommendations. Vaccine. (2015) 33:43834. doi: $10.1016 /$ j.vaccine.2014.12.002

67. Harper DM, DeMars LR. HPV vaccines - a review of the first decade. Gynecol Oncol. (2017) 146:196-204. doi: 10.1016/j.ygyno.2017.04.004

68. Kim JJ, Burger EA, Sy S, Campos NG. Optimal cervical cancer screening in women vaccinated against human papillomavirus. J Natl Cancer Inst. (2017) 109:djw216. doi: 10.1093/jnci/djw216

69. Vekemans J, Brennan MJ, Hatherill M, Schrager L, Fritzell B, Rutkowski $\mathrm{K}$, et al. Preferred product characteristics for therapeutic vaccines to improve tuberculosis treatment outcomes: key considerations from World Health Organization consultations. Vaccine. (2020) 38:135-42. doi: 10.1016/j.vaccine.2019.10.072

70. Bruni L, Diaz M, Barrionuevo-Rosas L, Herrero R, Bray F, Bosch FX, et al. Global estimates of human papillomavirus vaccination coverage by region and income level: a pooled analysis. Lancet Glob Health. (2016) 4:e45363. doi: 10.1016/S2214-109X(16)30099-7

71. Doherty M, Buchy P, Standaert B, Giaquinto C, Prado-Cohrs D. Vaccine impact: Benefits for human health. Vaccine. (2016) 34:6707-14. doi: 10.1016/j.vaccine.2016.10.025

72. Clendinen CS, Stupp GS, Ajredini R, Lee-McMullen B, Beecher C, Edison AS. Manufacturing costs of HPV vaccines for developing countries. Vaccine. (2016) 34:5984-9. doi: 10.1016/j.vaccine.2016.09.042

73. Bosch FX, Robles C, Díaz M, Arbyn M, Baussano I, Clavel C, et al. HPVFASTER: broadening the scope for prevention of HPV-related cancer. Nat Rev Clin Oncol. (2016) 13:119-32. doi: 10.1038/nrclinonc.2015.146

74. Kalinowski P, Grzadziel A. HPV vaccinations in lublin region, poland. Postepy Hig Med Dosw. (2017) 71:92-7. doi: 10.5604/01.3001.0010.3793

75. Restivo V, Costantino C, Fazio TF, Casuccio N, D'Angelo C, Vitale F, et al. Factors associated with HPV vaccine refusal among young adult women after ten years of vaccine implementation. Int J Environ Res Public Health. (2018) 15:770. doi: 10.3390/ijerph15040770

76. Tjalma W, Brasseur C, Top G, Ribesse N, Morales I, Van Damme PA. HPV vaccination coverage in the federal state of belgium according to regions and their impact. Facts Views Vis Obgyn. (2018) 10:101-5.

77. So VHT, Channon AA, Ali MM, Merdad L, Al Sabahi S, Al Suwaidi $\mathrm{H}$, et al. Uptake of breast and cervical cancer screening in four Gulf Cooperation Council countries. Eur J Cancer Prev. (2019) 28:4516. doi: $10.1097 / C E J .0000000000000466$

78. Hall MT, Simms KT, Lew JB, Smith MA, Brotherton JM, Saville $\mathrm{M}$, et al. The projected timeframe until cervical cancer elimination in Australia: a modelling study. Lancet Public Health. (2019) 4:e1927. doi: $10.1016 / \mathrm{S} 2468-2667(18) 30183-\mathrm{X}$

79. Simms KT, Steinberg J, Caruana M, Smith MA, Lew JB, Soerjomataram I, et al. Impact of scaled up human papillomavirus vaccination and cervical screening and the potential for global elimination of cervical cancer in 181 countries, 2020-99: a modelling study. Lancet Oncol. (2019) 20:394407. doi: 10.1016/S1470-2045(18)30836-2

80. Machalek DA, Chow EP, Garland SM, Wigan R, Cornall AM, Fairley CK, et al. Human papillomavirus prevalence in unvaccinated heterosexual men 
after a national female vaccination program. J Infect Dis. (2017) 215:2028. doi: 10.1093/infdis/jiw530

81. Chen W, Zheng R, Baade PD, Zhang S, Zeng H, Bray F, et al. Cancer statistics in China, 2015. CA Cancer J Clin. (2016) 66:115-32. doi: 10.3322/caac.21338

82. Li X, Zheng R, Li X, Shan H, Wu Q, Wang Y, et al. Trends of incidence rate and age at diagnosis for cervical cancer in China, from 2000 to 2014. Chin J Cancer Res. (2017) 29:477-86. doi: 10.21147/j.issn.1000-9604.2017.06.02

83. Li X, Deng Y, Tang W, Sun Q, Chen Y, Yang C, et al. Urban-rural disparity in cancer incidence, mortality, and survivals in shanghai, china, during 2002 and 2015. Front Oncol. (2018) 8:579. doi: 10.3389/fonc.2018.00579

84. Zhu FC, Hu SY, Hong Y, Hu YM, Zhang X, Zhang YJ, et al. Efficacy, immunogenicity and safety of the AS04-HPV-16/18 vaccine in Chinese women aged 18-25 years: end-of-study results from a phase II/III, randomised, controlled trial. Cancer Med. (2019) 8:6195-211. doi: 10.1002/cam4.2399

85. Wei L, Xie X, Liu J, Zhao Y, Chen W, Zhao C, et al. Efficacy of quadrivalent human papillomavirus vaccine against persistent infection and genital disease in Chinese women: a randomized, placebo-controlled trial with 78-month follow-up. Vaccine. (2019) 37:3617-24. doi: 10.1016/j.vaccine.2018.08.009

86. Bao YP, Li N, Smith JS, Qiao YL, members A. Human papillomavirus type distribution in women from Asia: a meta-analysis. Int J Gynecol Cancer. (2008) 18:71-9. doi: 10.1111/j.1525-1438.2007.00959.x

87. Briones R, Nan X, Madden K, Waks L. When vaccines go viral: an analysis of HPV vaccine coverage on YouTube. Health Commun. (2012) 27:47885. doi: $10.1080 / 10410236.2011 .610258$
88. Dyer O. Canadian academic's call for moratorium on HPV vaccine sparks controversy. BMJ. (2015) 351:h5692. doi: 10.1136/bmj.h5692

89. Spencer JC, Brewer NT, Trogdon JG, Wheeler SB, Dusetzina SB. Predictors of human papillomavirus vaccine follow-through among privately insured US patients. Am J Public Health. (2018) 108:946-50. doi: 10.2105/AJPH.2018.304408

90. Perez S, Zimet GD, Tatar O, Stupiansky NW, Fisher WA, Rosberger Z. Human papillomavirus vaccines: successes and future challenges. Drugs. (2018) 78:1385-96. doi: 10.1007/s40265-018-0975-6

91. Rosberger Z, Steben M, Norris T, McFadyen A, Shapiro GK. A mixed twodose vaccination schedule: not enough evidence to support a policy change in Quebec. Vaccine. (2019) 37:4421. doi: 10.1016/j.vaccine.2019.03.083

Conflict of Interest: The authors declare that the research was conducted in the absence of any commercial or financial relationships that could be construed as a potential conflict of interest.

Copyright (๑) 2020 Zhou, Sun, Yao, Li, Wang and Lin. This is an open-access article distributed under the terms of the Creative Commons Attribution License (CC BY). The use, distribution or reproduction in other forums is permitted, provided the original author(s) and the copyright owner(s) are credited and that the original publication in this journal is cited, in accordance with accepted academic practice. No use, distribution or reproduction is permitted which does not comply with these terms. 